OPEN ACCESS

Edited by:

Cheng Zhong,

Tianiin University, China

Reviewed by:

Xifei Li,

Xi'an University of Technology, China

Shiyou Zheng,

University of Shanghai for Science and

Technology, China

*Correspondence:

Yuxin Tang

yxtang@um.edu.mo

Specialty section:

This article was submitted to

Electrochemistry

a section of the journal

Frontiers in Chemistry

Received: 12 December 2019 Accepted: 18 February 2020

Published: 28 February 2020

Citation:

Chen M, Zhang Y, Xing G and Tang Y (2020) Building High Power Density of Sodium-Ion Batteries: Importance of Multidimensional Diffusion Pathways in Cathode Materials.

Front. Chem. 8:152

doi: 10.3389/fchem.2020.00152

\section{Building High Power Density of Sodium-Ion Batteries: Importance of Multidimensional Diffusion Pathways in Cathode Materials}

\author{
Mingzhe Chen, Yanyan Zhang, Guichuan Xing and Yuxin Tang* \\ Institute of Applied Physics and Materials Engineering, University of Macau, Macau, China
}

Emerging sodium-ion batteries (SIBs) devices hold the promise to leapfrog over existing lithium-ion batteries technologies with respect to desirable power/energy densities and the abundant sodium sources on the earth. To this end, the discoveries on novel cathode materials with outstanding rate capabilities are being given high priority in the quest to achieve high power density SIBs devices, and the multi-dimensional $\mathrm{Na}^{+}$migration pathways with low diffusion energy barriers are crucial. In light of this, the recent development of Prussian blue analogs and sodium superionic conductor (NASICON)-type materials with 3D $\mathrm{Na}^{+}$diffusion pathways for building high power density NIBs are provided in this perspective. Ultimately, the future research directions to realize them for real applications are also discussed.

Keywords: high power density, multidimensional diffusion pathways, cathode materials, sodium-ion batteries devices, materials design

\section{INTRODUCTION}

Although Li-ion batteries have received tremendous success in electronic and portable devices, the high price of lithium due to its limited and unequally distributed resources (Figure 1A) may hinder its further application in novel large-scale electrochemical energy storages systems (EESs) (Choi and Aurbach, 2016; Hwang et al., 2017; Vaalma et al., 2018). The rapid development of renewable energy sources such as wind energy, solar energy, and tide energy requires more cost-efficient and highly reliable large-scale EESs. SIBs have been widely considered as promising candidates for next-generation low-cost energy storage devices due to the almost unlimited resources of sodium (Li et al., 2017; Chen et al., 2019b,c). Due to the large atomic radius of $\mathrm{Na}\left(0.97 \AA\right.$ for $\mathrm{Na}^{+}$and only $0.68 \AA$ for $\mathrm{Li}^{+}$), however, hurdles that hinder the further applications of SIBs are mainly found in their sluggish sodium diffusion kinetics and large volume expansion, which are both critical parameters for revolutionary power density performance that can meet the real needs of power grids and large-scale EESs (Dai et al., 2017; Chen et al., 2019a). Therefore, it is important to explore novel cathode materials for SIBs with improved Na kinetics. From the solid-state diffusion perspective, the dimensions of diffusion pathways are the extremely important factors determining the diffusion coefficient of $\mathrm{Na}^{+}$as well as the diffusion energy barriers. For facile and rapid ionic transportation, the cathode materials that possess three-dimensional (3D) $\mathrm{Na}^{+}$migration pathways accompanied by low diffusion energy barriers are promising for building the high-power density SIBs (You et al., 2016; Chen S. et al., 2017). To this end, two emerging groups of Prussian blue analogs (PBAs) and sodium superionic conductor (NASICON)-type materials are recently 


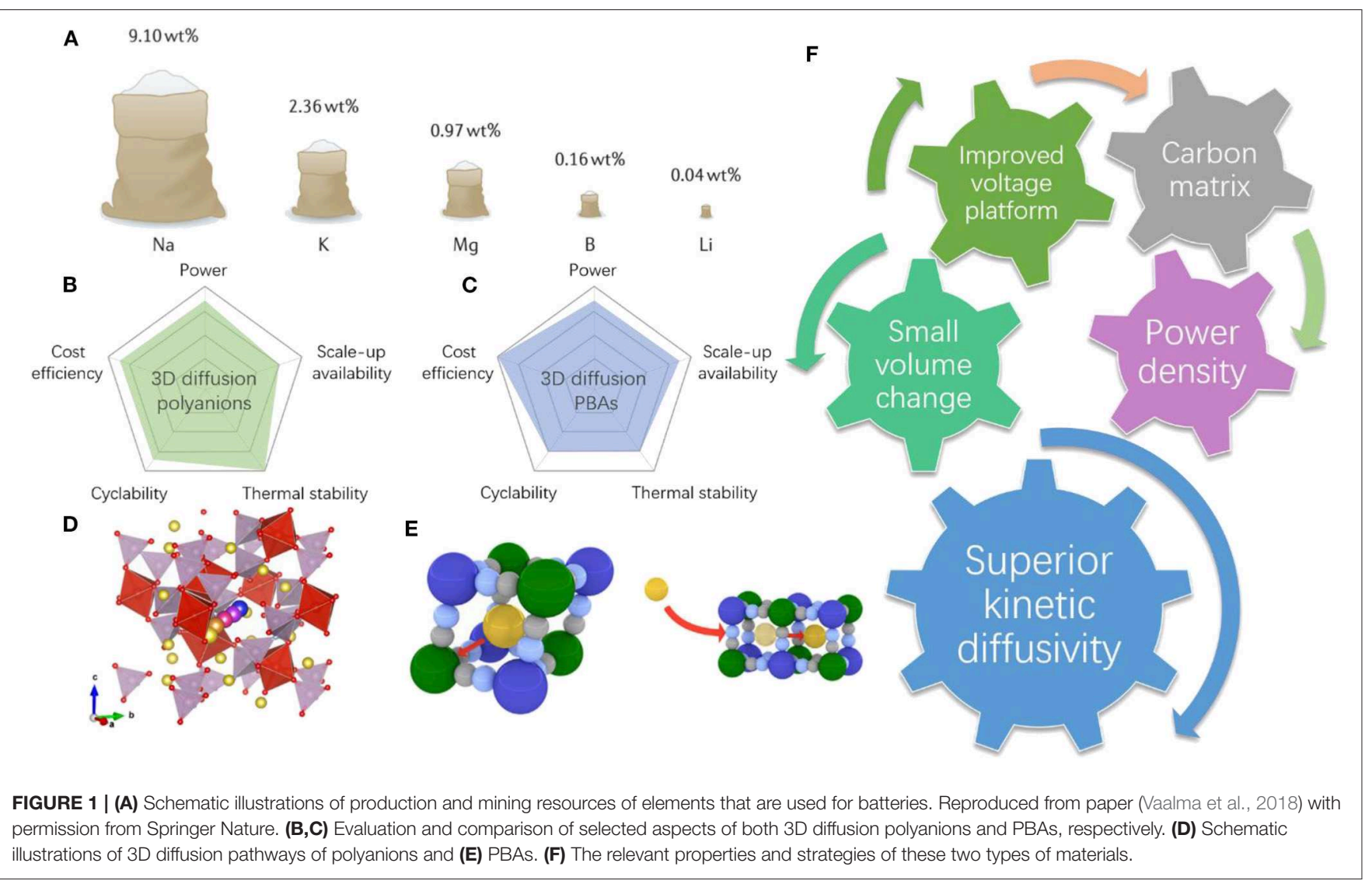

developed. They both possess open 3D stable frameworks, exceptionally high ionic conductivities, and 3D sodium diffusion pathways. More importantly, these two types of materials usually undergo minimal volume changes during cycling, and their structures are highly tunable with different electrochemical properties (Lee et al., 2014; Wang B. et al., 2018). We compared the five important aspects for the real applications of these two types of materials in Figures 1B-E. We have witnessed an outstanding increase in publications regarding cathode materials with 3D diffusion pathways (Kim et al., 2012; You et al., 2016; Fedotov et al., 2018; Hurlbutt et al., 2018). Specifically, strategies such us nanosizing particles, introducing a uniform carbon coating/carbon matrix, and heteroatom doping are heavily used for better electrochemical properties (Figure 1F). These strategies have been developed to regulate the microstructures and surface atomic configurations to accelerate the charge transfer and kinetic properties as the same time.

In this perspective, we highlight (i) the specific key issues that are hindering the wider applications of these $3 \mathrm{D} \mathrm{Na}^{+}$diffusion pathway materials, (ii) the recent novel and potential strategies to achieve high-performance SIBs, and (iii) the approaches to achieve higher energy/power densities and long-term cycling stability, and (iv) the new emerging SIB devices. Our perspective sharps the future development of cathode materials for the highpower density of SIBs and provides effective methodologies for other related next-generation energy storage devices.

\section{PBAs WITH 3D $\mathrm{Na}^{+}$DIFFUSION PATHWAYS}

Low-cost Na-hosting Prussian blue analogs usually possess the general structural formula of $\mathrm{Na}_{x} \mathrm{M}_{2}\left[\mathrm{M}_{1}(\mathrm{CN})_{6}\right]_{y} \cdot n \mathrm{H}_{2} \mathrm{O}(0 \leq x \leq$ $2,0 \leq y \leq 1)$, where $\mathrm{M}_{1}$ and $\mathrm{M}_{2}$ are transition metal such us $\mathrm{Mn}$, $\mathrm{Fe}, \mathrm{Co}$, etc. (Li et al., 2015). Within this structure, PBAs allow various modifications of their chemical compositions without changing the overall crystalline structure. Normally, most of the studied PBAs occurs as cubic phases with 6-fold coordination of $\mathrm{Fe}^{\mathrm{IIIIIII}}-\mathrm{O}_{6}$ octahedra participating in the redox reactions (Xiao et al., 2015). Theoretically, PBAs allow a maximum number of two $\mathrm{Na}^{+}$ions per formula unit if both the $\mathrm{M}_{1}$ and the $\mathrm{M}_{2}$ transition metal elements are electrochemically active, which can lead to a potential capacity over $170 \mathrm{mAh} \mathrm{g}^{-1}$, completely comparable with all other kinds of cathode material for SIBs (Wu et al., 2017). Such high capacity is not easy to achieve, however, since the defects, vacancies, and water molecules will greatly influence the crystal structures of PBAs. Their zeolitic water molecules tend to occupy the interstitial sites that are essential for the sodium ion diffusion. The ideal situation is that all the zeolitic water can be completely removed, while the coordination water can be retained to render structural stability. In addition, the poor intrinsic electronic conductivity of PBAs also remains a great challenge for better C-rate performance, although they possess 3D diffusion pathways. From the previous density function theory (DFT) calculations, the intercalation of $\mathrm{Na}^{+}$with a larger ionic radius will incur the cost of increased insertion 
potential, since the steric interactions have a close correlation with the hydration energy. Both sodium and potassium possess sufficiently high redox potentials that an appropriate energy density can be secured. The role of water in ionic conductivity is still an open question for all researchers. A paddlewheel mechanism was previously proposed, in which the alkali metal ions move through channels and vacancies (Wessells et al., 2011). Coordinated water is beneficial to facilitate ion migration, while zeolitic water has a negative influence, obstructing improvement of ionic conductivity. Both computational and experimental insights into the effects of the presence of water in PBAs are urgently needed.

One promising approach is to synthesize and optimize the Na-rich phase, Prussian white. When $\mathrm{M}_{2}$ is $\mathrm{Mn}$ and $\mathrm{M}_{1}$ is $\mathrm{Fe}$ in $\mathrm{Na}_{x} \mathrm{M}_{2}\left[\mathrm{M}_{1}(\mathrm{CN})_{6}\right]_{y} \cdot n \mathrm{H}_{2} \mathrm{O}(0 \leq x \leq 2,0 \leq y \leq 1)$, a higher content of $\mathrm{Na}$ can be achieved. The valence of $\mathrm{Fe}$ is +2 , however, and it is vulnerable to being oxidized to +3 , so an inert atmosphere is required during both fabrication and centrifugal separation processes. By facile sodium citrate added precipitation method, Na-rich monoclinic phase $\mathrm{Na}_{x} \mathrm{MnFe}(\mathrm{CN})_{6}$ was successfully synthesized by Shen et al. (2018). Uniform microsize cuboid particles were obtained, and all the involved elements were uniformly distributed (Figures 2A-C). The high reversible capacity of $133.1 \mathrm{mAh}$ $\mathrm{g}^{-1}$ was obtained, and satisfactory rate performance was achieved from $0.1 \mathrm{C}$ to $10 \mathrm{C}\left(1 \mathrm{C}=150 \mathrm{~mA} \mathrm{~g}^{-1}\right)$, as shown in Figures 2D,E. The uniform cuboid particle morphology provides extra convenience for fast ion diffusion apart from the original $3 \mathrm{D}$ pathways. In recent years, rhombohedral PBAs have been receiving more and more attention, since the concentration of coordinated water can be reduced due to the rhombohedral lattice symmetry, which is particularly favorable for the Na-rich phases. A zero-strain nickel hexacyanoferrate (NiHCF) was introduced by Ji et al. (2016) using a simple coprecipitation method. It delivered a high operation voltage, good cycling performance, and superior C-rate capability. They found that the unexpected high operation voltage was mainly attributable to the asymmetric residence of $\mathrm{Na}^{+}$ions along with the low charge density around $\mathrm{Fe}^{2+}$. Different charge density analyses indicated that the $\mathrm{Na}^{+}$ ions in cubic-structured NiHCF (c-NiHCF) tend to stay exactly in the center of interstitial channels (Figure 2F), while for rhombohedral-structured NiHCF (r-NiHCF), the $\mathrm{Na}^{+}$ ions prefer to asymmetrically stay at $\mathrm{N}$-coordinated corners (Figure 2G). Also the Na-N distance in $\mathrm{r}-\mathrm{NiHCF}$ is shorter than that in $\mathrm{c}-\mathrm{NiHCF}$. Dramatic electron polarization round $\mathrm{N}$ atoms occurs, leading to a charge redistribution between adjacent $\mathrm{Ni}^{2+}$ and $\mathrm{Fe}^{2+}$ ions.

Therefore, the operation voltage of $\mathrm{r}-\mathrm{NiHCF}$ is slightly higher than that of $\mathrm{c}-\mathrm{NiHCF}$. This phenomenon also facilitates fast sodium diffusion with excellent C-rate performances. In addition, the open-channel $3 \mathrm{D}$ diffusion pathways can guarantee satisfactory low-temperature performance. You et al. synthesized the $\mathrm{Na}_{2} \mathrm{FeFe}(\mathrm{CN})_{6}$ materials with carbon nanotubes (CNT) to achieve potential low-temperature properties (You et al., 2016). The well-decorated CNT network provides excellent electrical contact with the current collector. In addition, they performed a DFT study and found that the evenly distributed enriched $24 d$ sites can provide uniform $3 \mathrm{D} \mathrm{Na}{ }^{+}$diffusion and feasible diffusion pathways, as shown in Figures 2H,I. There, PBAs are ideal candidates for low-temperature SIBs (Song et al., 2015; Peng et al., 2018). Further exploration should be focused on the inherent relationship between the crystal structure and the vacancies and water, and how these factors influence the sodium diffusion under various circumstances also needs to be urgently understood in detail.

\section{POLYANIONIC COMPOUNDS WITH 3D Na+ DIFFUSION PATHWAYS}

The polyanionic-type cathode materials are one of the important branches among all the kinds of available electrodes for SIBs. Their merits are obvious and distinctive, such as outstanding cycling stability, high safety, and suitable operating voltages (Lu et al., 2017; Chen et al., 2020). Most of the polyanionic compounds consist of the corner- or edge-sharing $\mathrm{MO}_{6}(M$ $=$ transition metal) octahedra with connected $\mathrm{XO}_{4}(X=$ $\mathrm{P}, \mathrm{S}, \mathrm{Si}$, etc.) tetrahedra. This unique structure can provide sufficient stability and ionicity around the $\mathrm{MO}_{6}$ octahedra, and, in turn, the ionic conductivity can be sustained within its 3D framework (Guo et al., 2017). Their sturdy framework can also render only small or even tiny volume shrinkage during cycling, since the topotactic reaction mechanism is dominant. Most of the polyanionic compounds are thermally stable due to their non-flammable nature (Ni et al., 2017). Also, polyanionic compounds normally possess high initial cycle Coulombic efficiency (ICE), which is an important parameter when making full cells. Nevertheless, most of the polyanionic compounds suffer from inherent low electronic conductivity, and therefore a well-decorated carbon coating and network are essential for their C-rate performance. According to the previous report, the majority of polyanionic composites possess one- or two-dimensional (1D or 2D) sodium diffusion pathways (Kim et al., 2013, 2015; Chen M. et al., 2017; Panigrahi et al., 2017; Chen et al., 2018; Fang et al., 2018; Zhu et al., 2019). The low-dimensional sodium diffusion pathways cannot guarantee satisfactory C-rate capabilities, especially in low temperature environments. Therefore, it is necessary to highlight those polyanionic compounds that possess unique 3D sodium diffusion pathways that are conducive toward real applications of SIBs and relevant energy storage devices. One class of these polyanionic composites comprises the sodium superionic conductor (NASICON)-type materials. Due to their high ionic conductivities, the NASICON-type materials also have been investigated as solid-state electrolytes (Li et al., 2018; Lu et al., 2018). Numerous papers have reported various NASICON-type materials, including both cathodes and anodes for SIB devices (Bui et al., 2015; Wang et al., 2017; Gao et al., 2018; Hu et al., 2018; Wang E. et al., 2018). Recently, we have introduced a new Fe-based polyanionic compound, $\mathrm{Na}_{4} \mathrm{Fe}_{3}\left(\mathrm{PO}_{4}\right)_{2}\left(\mathrm{P}_{2} \mathrm{O}_{7}\right)$, which can be classified as a new NASICON-type material (Chen et al., 2019a). With the help of ethylenediaminetetraacetic acid (EDTA) as the complexing agent in a sol-gel approach, the 

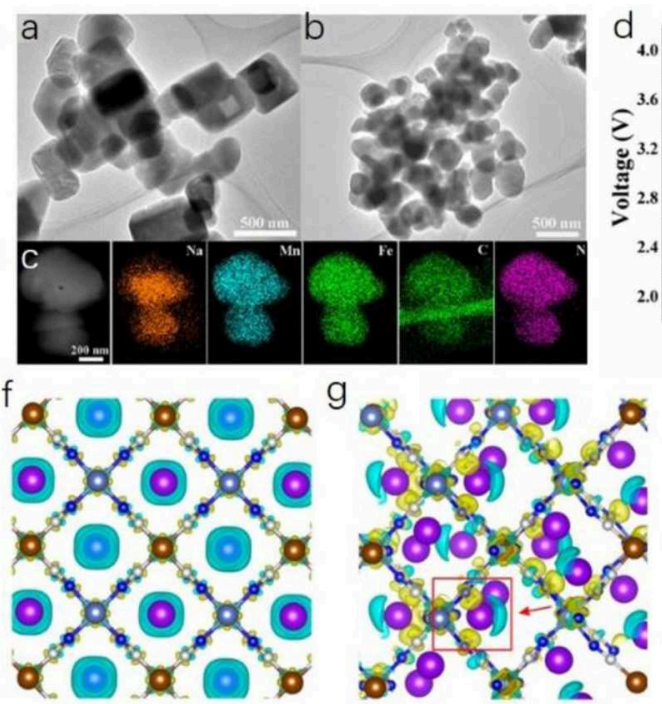

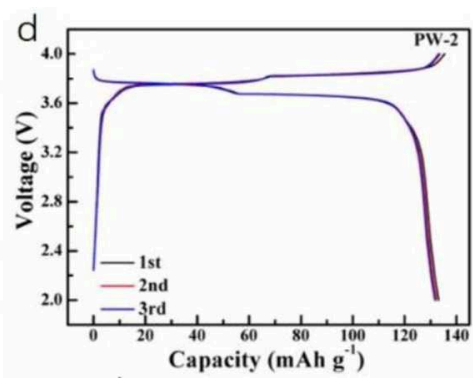

h oC on $\odot \mathrm{Fe} \odot \mathrm{Ni} \odot \mathrm{Na}$
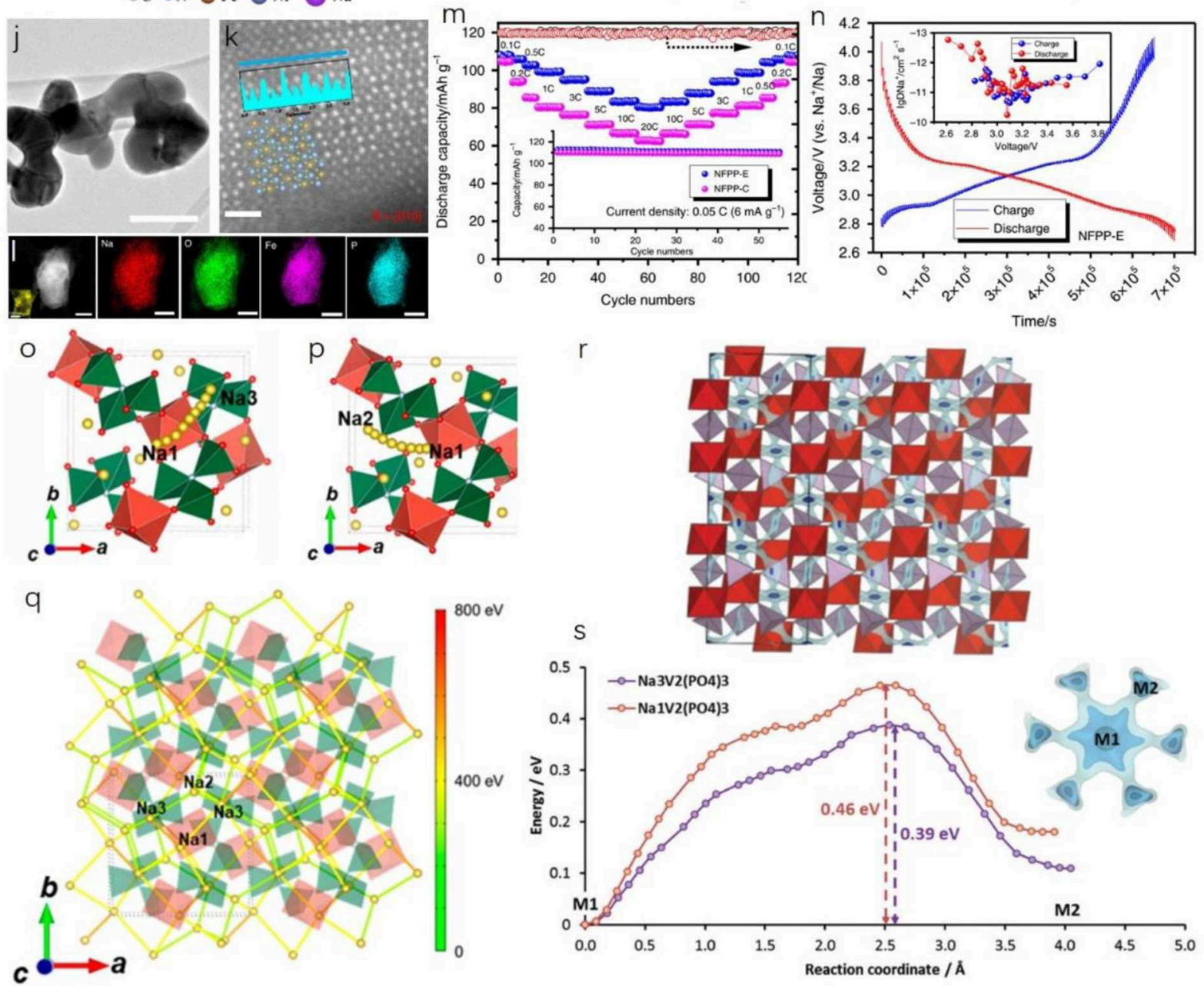

FIGURE 2 | (A,B) Transmission electron microscope (TEM) images of Na-rich monoclinic phase NaxMnFe(CN)6. (C) Scanning TEM-energy dispersive spectroscopy (STEM-EDS) element mapping results for $\mathrm{Na}_{x} \mathrm{MnFe}(\mathrm{CN})_{6}$. (D) Charge-discharge profile and (E) C-rate performance of the as-obtained sample. Reproduced from paper (Shen et al., 2018) with permission from the American Chemical Society. Charge density analyses of (F) cubic-structured NiHCF and (G) rhombohedral-structured NiHCF. Reproduced from paper (Ji et al., 2016) with permission from the American Chemical Society. (H,I) Front view of possible 3D Na+ pathways between adjacent equivalent 24d sites. Reproduced from paper (You et al., 2016) with permission from WILEY-VCH. (J) Bright field (BF) image, 
FIGURE 2 | (K) High-angle annular dark-field (HAADF) image with line profile in the inset, and (L) STEM-EDS mapping of as-obtained $\mathrm{Na}_{4} \mathrm{Fe}_{3}\left(\mathrm{PO}_{4}\right)_{2}\left(\mathrm{P}_{2} \mathrm{O}_{7}\right)(\mathrm{NFPP})$ nanoplate. (M) C-rate performance and cycling stability (inset) of NFPP-E nanoplate. (N) Galvanostatic intermittent titration technique (GITT) curve of NFPP-E electrode and corresponding calculated diffusion coefficient of $\mathrm{Na}^{+}$ions. Reproduced from paper (Chen et al., 2019a) with permission from the Nature Publishing group. (O,P) Two typical 3D sodium diffusion pathways in $\mathrm{Na}_{3} \mathrm{~V}\left(\mathrm{PO}_{3}\right)_{3} \mathrm{~N}$ material and the $\mathbf{( Q )}$ corresponding activation barriers. Reproduced from paper (Kim et al., 2017) with permission from the American Chemical Society. (R) NASICON-type $\mathrm{Na}_{3} \mathrm{~V}_{2}\left(\mathrm{PO}_{4}\right)_{3}$ material with energy isosurface, showing interweaving of $3 \mathrm{D}$ Na ${ }^{+}$ pathways and (S) migration pathways of $\mathrm{Na}^{+}$in the open 3D framework involving M1-M2-M1 hopping. Reproduced from paper (Wong et al., 2017) with permission from the Royal Society of Chemistry.

$\mathrm{Na}_{4} \mathrm{Fe}_{3}\left(\mathrm{PO}_{4}\right)_{2}\left(\mathrm{P}_{2} \mathrm{O}_{7}\right)$ nanoplates (denoted as NPFF-E) can be well-obtained with a high degree of crystallinity, as shown in Figures 2J,K. All the detected elements are uniformly distributed in one NPFF-E particle (Figure 2L). Outstanding C-rate performance can be obtained, and satisfactory cycling stability can be achieved as well (Figure $\mathbf{2 M}$ ). In addition, we also determined the sodium diffusion coefficient through a galvanostatic intermittent titration technique (GITT) study. From Figure 2N, the sodium diffusion coefficients range from $10^{-13}$ to $10^{-10} \mathrm{~cm}^{2} \mathrm{~s}^{-1}$, which is highly competitive with other recognized NASICON-type materials. These high diffusion coefficients are critical for low-temperature performance.

Another newly recognized family of NASICON-type cathode materials with $3 \mathrm{D}$ sodium diffusion pathways comprises the $\mathrm{V}$ based $\mathrm{N}$-substituted polyanionic materials. Kang et al. recently reported a new member of the $4 \mathrm{~V}$-class of zero-strain cathode materials, $\mathrm{Na}_{3} \mathrm{~V}\left(\mathrm{PO}_{3}\right)_{3} \mathrm{~N}$, with unique $3 \mathrm{D}$ diffusion pathways for $\mathrm{Na}^{+}$ions (Kim et al., 2017). One $\mathrm{PO}_{3} \mathrm{~N}$ tetrahedron is connected to three $\mathrm{VO}_{6}$ octahedra, leading to a strong inductive effect which, in turn, influences the operation voltage during charge/discharge. The relevant sodium ion diffusion pathways and diffusion energy barriers were calculated via a DFT study, and illustrations are presented in Figures 2O-Q. This exciting discovery has opened up new interest in the highvoltage $\mathrm{N}$-substituted cathode materials with unique unexpected $3 \mathrm{D}$ sodium diffusion pathways. As for the well-recognized NASICON-type $\mathrm{Na}_{3} \mathrm{~V}_{2}\left(\mathrm{PO}_{4}\right)_{3}$ material, great attention has been extensively paid to it due to its outstanding merits (high operation voltage, acceptable specific capacity, and excellent cyclability). Its $3 \mathrm{D}$ sodium diffusion properties have been well-investigated, with the 3D network involving M1-M2M1 hops and low energy barriers. Each $\mathrm{Na}$ in an M1 site is actively connected to six M2 sites. The corresponding illustrations are shown in Figures 2R,S. In addition, we would like to see more polyanionic compounds discovered with $3 \mathrm{D} \mathrm{Na}^{+}$diffusion pathways in the search for advanced SIB storage devices with high power densities. It is suggested that further exploration should be concentrated on improving the reaction kinetics based on a more comprehensive understanding. Nevertheless, due to the intrinsic low electron conductivity of polyanionic material, how to maintain the well-designed carbon matrix or network and large-scale synthesis is still very challenging.

\section{DISCUSSION AND PERSPECTIVES}

The ultimate goal for advanced sodium ion battery devices has never changed, that they should be thoroughly competitive with the lithium ion battery in energy density, power density, and lower overall manufacturing cost (Tang et al., 2017, 2018; Zhang et al., 2017, 2019; Guo et al., 2020). For fullcell sodium ion battery devices, the emphasis always falls on the cathode parts, since they possess key parameters such as high enough operation voltages, large theoretical capacity, and most of all, acceptable high-rate capability. Materials with 3D sodium diffusion pathways are a promising branch among all the reported ones, and their distinctive physical and chemical properties deserve more integrated investigations and a deeper and more comprehensive understanding. Both the PBAs and the polyanionic compounds with $3 \mathrm{D}$ sodium diffusion pathways have shown outstanding fast kinetics, leading to superior C-rate performance at various current densities. Further investigations are suggested to be focused on the improvements of their intrinsic kinetic properties as well as mass-production issues. Specifically, for PBAs, as mentioned above, the structurekinetics-performance relationship is subject to the influence of each crystallographic component: binary carbon- and nitrogencoordinated transition metal ions, the density distribution of electronic states at each sodium site, and the vacancies created by either zeolitic water or coordinated water. When dealing with the specific concerns about the actual $\mathrm{Na}^{+}$diffusion channels and ionic conductivity, the bond length between carbon and carbon-coordinated transition metal elements is one of the key parameters for channel size, which, in turn, affects the ionic conductivity. The content of vacancies also has a strong influence on the $\mathrm{Na}^{+}$diffusion channels and ionic conductivity. The appropriate amount of vacancies will provide extra channels for $\mathrm{Na}^{+}$diffusion without cracking the local crystal structure. Nevertheless, how these parameters quantitatively influence the ionic conductivity as well as the crystal stability is still poorly understood. Another important issue that should not be neglected is the electronic conductivity. Up until now, only a few papers have focused on improving electronic conductivity directly by using the generalized gradient approximation with the Hubbard correction (GGA + U) method. Experimentally speaking, the electronic conductivity is strongly correlated with all the factors mentioned above and needs to be determined in an equational relationship. In the case of polyanionic compounds, the electronic conductivity is the most troublesome problem, and a well-designed carbon coating and carbon network are necessary even when the materials have high ionic conductivities. Therefore, how to maintain the delicate carbon network in a large-scale manufacturing procedure is a critical issue for the real application of polyanionic composites. Unlike the PBAs, only some of the reported materials possess $3 \mathrm{D}$ sodium diffusion pathways, although almost 
all the polyanionic compounds have open 3D frameworks. Compared to the PBAs, the activation energy barriers of these polyanionic compounds are slightly higher. Heteroatom doping can be considered as an effective approach to alter the pristine electron state density distributions around both $\mathrm{MO}_{6}$ octahedra and $\mathrm{XO}_{4}$ tetrahedra, which, in turn, may lower the charge barriers around each sodium site. The transition metal migration is also poorly understood to date [such as for $\mathrm{Na}_{4} \mathrm{M}_{3}\left(\mathrm{PO}_{4}\right)_{2}\left(\mathrm{P}_{2} \mathrm{O}_{7}\right)(\mathrm{M}=\mathrm{Fe}, \mathrm{Mn}$, etc.)], and how the phenomenon affects the related ion diffusion pathways and ionic conductivity should be determined in the future. It is also important to continuously discover more novel polyanionic cathode materials with $3 \mathrm{D}$ sodium diffusion pathways that have higher capacity and operation voltages. Besides, another important research direction is that how to combine both the PBAs and polyanions with the solid-state electrolyte properly so that their unique $3 \mathrm{D}$ diffusion properties can be more significantly magnified.

In Table S1, we summarize some of the reported cathodes for SIB devices with unique 3D diffusion pathways and their corresponding diffusion coefficients and activation barriers. These types of cathodes are especially important for highpower SIB devices for real applications. We can reasonably anticipate more technical breakthroughs, such as precise control of the water content in PBAs, ways to maintain a welldecorated carbon network at the industrial level, efficient organic/solid state electrolytes to fully achieve their theoretical capacities, and, in turn, the advanced SIB devices can be further

\section{REFERENCES}

Bui, K. M., Dinh, V. A., Okada, S., and Ohno, T. (2015). Hybrid functional study of the NASICON-type $\mathrm{Na}_{3} \mathrm{~V}_{2}\left(\mathrm{PO}_{4}\right)_{3}$ : crystal and electronic structures, and polaron-Na vacancy complex diffusion. Phys. Chem. Chem. Phys. 17, 30433-30439. doi: 10.1039/C5CP05323D

Chen, M., Chen, L., Hu, Z., Liu, Q., Zhang, B., Hu, Y., et al. (2017). Carbon-Coated $\mathrm{Na}_{3.32} \mathrm{Fe}_{2.34}\left(\mathrm{P}_{2} \mathrm{O}_{7}\right)_{2}$ cathode material for high-rate and long-life sodium-ion batteries. Adv. Mater. 29:1605535. doi: 10.1002/adma.201605535

Chen, M., Cortie, D., Hu, Z., Jin, H., Wang, S., Gu, Q., et al. (2018). A novel graphene oxide wrapped $\mathrm{Na}_{2} \mathrm{Fe}_{2}\left(\mathrm{SO}_{4}\right)_{3} / \mathrm{C}$ cathode composite for long life and high energy density sodium-ion batteries. Adv. Energy Mater. 8:1800944. doi: $10.1002 /$ aenm.201800944

Chen, M., Hua, W., Xiao, J., Cortie, D., Chen, W., Wang, E., et al. (2019a). NASICON-type air-stable and all-climate cathode for sodium-ion batteries with low cost and high-power density. Nat. Commun. 10:1480. doi: 10.1038/s41467-019-09170-5

Chen, M., Liu, Q., Wang, S.-W., Wang, E., Guo, X., and Chou, S.-L. (2019b). High-abundance and low-cost metal-based cathode materials for sodiumion batteries: problems, progress, and key technologies. Adv. Energy Mater. 9:1803609. doi: 10.1002/aenm.201803609

Chen, M., Liu, Q., Zhang, Y., Xing, G., Chou, S. and Tang, Y. (2020). Emerging polyanionic and organic compounds for high energy density, non-aqueous potassium-ion batteries. J Mater. Chem. A. doi: 10.1039/C9TA11221A

Chen, M., Wang, E., Liu, Q., Guo, X., Chen, W., Chou, S.-L., et al. (2019c). Recent progress on iron- and manganese-based anodes for sodiumion and potassium-ion batteries. Energy Storage Mater. 19, 163-178. doi: 10.1016/j.ensm.2019.03.030

Chen, S., Wu, C., Shen, L., Zhu, C., Huang, Y., Xi, K., et al. (2017). Challenges and perspectives for NASICON-type electrode materials for advanced sodium-ion batteries. Adv. Mater. 29:1700431. doi: 10.1002/adma.201700431 designed and developed from a more technology based point of view.

\section{DATA AVAILABILITY STATEMENT}

The datasets generated for this study are available on request to the corresponding author.

\section{AUTHOR CONTRIBUTIONS}

MC, YZ, and GX prepared the manuscript. YT supervised the project.

\section{FUNDING}

This work was funded by The Science and Technology Development Fund, Macau SAR (Grant Nos. 0057/2019/A1 and 0092/2018/A2).

\section{ACKNOWLEDGMENTS}

The authors thank Dr. Tania Silver for critical reading of the manuscript.

\section{SUPPLEMENTARY MATERIAL}

The Supplementary Material for this article can be found online at: https://www.frontiersin.org/articles/10.3389/fchem. 2020.00152/full\#supplementary-material
Choi, J. W., and Aurbach, D. (2016). Promise and reality of post-lithiumion batteries with high energy densities. Nat. Rev. Mater. 1:16013. doi: 10.1038/natrevmats.2016.13

Dai, Z., Mani, U., Tan, H. T., and Yan, Q. (2017). Advanced cathode materials for sodium-ion batteries: what determines our choices? Small Methods 1:1700098. doi: 10.1002/smtd.201700098

Fang, Y., Liu, Q., Xiao, L., Rong, Y., Liu, Y., Chen, Z., et al. (2018). A fully sodiated $\mathrm{NaVOPO}_{4}$ with layered structure for high-voltage and longlifespan sodium-ion batteries. Chem 4, 1167-1180. doi: 10.1016/j.chempr.2018. 03.006

Fedotov, S. S., Kabanova, N. A., Kabanov, A. A., Blatov, V. A., Khasanova, N. R., and Antipov, E. V. (2018). Crystallochemical tools in the search for cathode materials of rechargeable $\mathrm{Na}$-ion batteries and analysis of their transport properties. Solid State Ion. 314, 129-140. doi: 10.1016/j.ssi.2017.11.008

Gao, H., Seymour, I. D., Xin, S., Xue, L., Henkelman, G., and Goodenough, J. B. (2018). $\mathrm{Na}_{3} \mathrm{MnZr}\left(\mathrm{PO}_{4}\right)_{3}$ : a high-voltage cathode for sodium batteries. J. Am. Chem. Soc. 140, 18192-18199. doi: 10.1021/jacs.8b11388

Guo, Q., Mao, J., Huang, J., Wang, Z., Zhang, Y., Hu, J., Dong, J., Sathasivam, S., Zhao, Y., Xing, G., et al. (2020). Reducing oxygen evolution reaction overpotential in cobalt-based electrocatalysts via optimizing the “microparticles-in-spider web” electrode configurations. Small 16:e1907029. doi: 10.1002/smll.201907029

Guo, S.-P., Li, J.-C., Xu, Q.-T., Ma, Z., and Xue, H.-G. (2017). Recent achievements on polyanion-type compounds for sodium-ion batteries: syntheses, crystal chemistry and electrochemical performance. J. Power Sources 361, 285-299. doi: 10.1016/j.jpowsour.2017.07.002

$\mathrm{Hu}$, Q., Yu, M., Liao, J., Wen, Z., and Chen, C. (2018). Porous carbon-coated $\mathrm{NaTi}_{2}\left(\mathrm{PO}_{4}\right)_{3}$ with superior rate and low-temperature properties. J Mater. Chem. A. 6, 2365-2370. doi: 10.1039/C7TA10207K

Hurlbutt, K., Wheeler, S., Capone, I., and Pasta, M. (2018). Prussian blue analogs as battery materials. Joule 2, 1950-1960. doi: 10.1016/j.joule.2018.07.017 
Hwang, J. Y., Myung, S. T., and Sun, Y. K. (2017). Sodium-ion batteries: present and future. Chem. Soc. Rev. 46, 3529-3614. doi: 10.1039/C6CS0 $0776 \mathrm{G}$

Ji, Z., Han, B., Liang, H., Zhou, C., Gao, Q., Xia, K., et al. (2016). On the mechanism of the improved operation voltage of rhombohedral nickel hexacyanoferrate as cathodes for sodium-ion batteries. ACS Appl. Mater. Interfaces 8, 33619-33625. doi: 10.1021/acsami.6b11070

Kim, H., Park, I., Seo, D. H., Lee, S., Kim, S. W., Kwon, W. J., et al. (2012). New iron-based mixed-polyanion cathodes for lithium and sodium rechargeable batteries: combined first principles calculations and experimental study. J. Am. Chem. Soc. 134, 10369-10372. doi: 10.1021/ja3038646

Kim, H., Shakoor, R. A., Park, C., Lim, S. Y., Kim, J.-S., Jo, Y. N., et al. (2013). $\mathrm{Na}_{2} \mathrm{FeP}_{2} \mathrm{O}_{7}$ as a promising iron-based pyrophosphate cathode for sodium rechargeable batteries: a combined experimental and theoretical study. $A d v$. Funct. Mater 23, 1147-1155. doi: 10.1002/adfm.201201589

Kim, H., Yoon, G., Park, I., Park, K.-Y., Lee, B., Kim, J., et al. (2015). Anomalous Jahn-Teller behavior in a manganese-based mixed-phosphate cathode for sodium ion batteries. Energy Environ. Sci. 8, 3325-3335. doi: 10.1039/C5EE01876E

Kim, J., Yoon, G., Lee, M. H., Kim, H., Lee, S., and Kang, K. (2017). New 4VClass and zero-strain cathode material for Na-ion batteries. Chem. Mater. 29, 7826-7832. doi: 10.1021/acs.chemmater.7b02477

Lee, H.-W., Wang, R. Y., Pasta, M., Lee, S. W., Liu, N., and Cui, Y. (2014). Manganese hexacyanomanganate open framework as a high-capacity positive electrode material for sodium-ion batteries. Nat. Commun. 5, 5280. doi: $10.1038 /$ ncomms6280

Li, W.-J., Chou, S.-L., Wang, J.-Z., Kang, Y.-M., Wang, J.-L., Liu, Y., et al. (2015). Facile method to synthesize Na-enriched $\mathrm{Na}_{1+x} \mathrm{FeFe}(\mathrm{CN})_{6}$ frameworks as cathode with superior electrochemical performance for sodium-ion batteries. Chem. Mater. 27, 1997-2003. doi: 10.1021/cm504091z

Li, X., Huang, Y., Wang, J., Miao, L., Li, Y., Liu, Y., et al. (2018). High valence Mo-doped $\mathrm{Na}_{3} \mathrm{~V}_{2}\left(\mathrm{PO}_{4}\right)_{3} / \mathrm{C}$ as a high rate and stable cycle-life cathode for sodium battery. J. Mater. Chem. A. 6, 1390-1396. doi: 10.1039/C7TA0 $8970 \mathrm{H}$

Li, Y., Lu, Y., Zhao, C., Hu, Y.-S., Titirici, M.-M., Li, H., et al. (2017). Recent advances of electrode materials for low-cost sodium-ion batteries toward practical application for grid energy storage. Energy Storage Mater. 7, 130-151. doi: 10.1016/j.ensm.2017.01.002

Lu, J., Nishimura, S.-,i., and Yamada, A. (2017). Polyanionic solid-solution cathodes for rechargeable batteries. Chem. Mater. 29, 3597-3602. doi: 10.1021/acs.chemmater.7b00226

Lu, Y., Li, L., Zhang, Q., Niu, Z., and Chen, J. (2018). Electrolyte and interface engineering for solid-state sodium batteries. Joule 2, 1747-1770. doi: 10.1016/j.joule.2018.07.028

Ni, Q., Bai, Y., Wu, F., and Wu, C. (2017). Polyanion-Type electrode materials for sodium-ion batteries. Adv. Sci. 4, 1600275. doi: 10.1002/advs.201600275

Panigrahi, A., Nishimura, S.-,I., Shimada, T., Watanabe, E., Zhao, W., Oyama, G., et al. (2017). Sodium Iron(II) Pyrosilicate $\mathrm{Na}_{2} \mathrm{Fe}_{2} \mathrm{Si}_{2} \mathrm{O}_{7}$ : a potential cathode material in the $\mathrm{Na}_{2} \mathrm{O}-\mathrm{FeO}-\mathrm{SiO}_{2}$ system. Chem. Mater. 29, 4361-4366. doi: 10.1021/acs.chemmater.7b00764

Peng, J., Wang, J., Yi, H., Hu, W., Yu, Y., Yin, J., et al. (2018). A Dual-Insertion type sodium-ion full cell based on high-quality ternary-metal prussian blue analogs. Adv. Energy Mater. 8:1702856. doi: 10.1002/aenm.201702856

Shen, Z., Guo, S., Liu, C., Sun, Y., Chen, Z., Tu, J., et al. (2018). Na-rich prussian white cathodes for long-life sodium-ion batteries. ACS Sustain. Chem. Eng. 6, 16121-16129. doi: 10.1021/acssuschemeng.8b02758

Song, J., Wang, L., Lu, Y., Liu, J., Guo, B., Xiao, P., et al. (2015). Removal of interstitial $\mathrm{H}_{2} \mathrm{O}$ in hexacyanometallates for a superior cathode of a sodium-ion battery. J Am. Chem. Soc. 137, 2658-2664. doi: 10.1021/ja51 $2383 b$

Tang, Y., Deng, J., Li, W., Malyi, O. I., Zhang, Y., Zhou, X., et al. (2017). Water-soluble sericin protein enabling stable solid-electrolyte interphase for fast charging high voltage battery electrode. Adv. Mater 29:1701828. doi: 10.1002/adma.201701828

Tang, Y., Zhang, Y., Malyi, O. I., Bucher, N., Xia, H., Xi, S., et al. (2018). Identifying the origin and contribution of surface storage in tio2(b) nanotube electrode by in situ dynamic valence state monitoring. Adv. Mater 30, 1802200. doi: 10.1002/adma.201802200

Vaalma, C., Buchholz, D., Weil, M., and Passerini, S. (2018). A cost and resource analysis of sodium-ion batteries. Nat. Rev. Mater. 3:18013. doi: $10.1038 /$ natrevmats. 2018.13

Wang, B., Han, Y., Wang, X., Bahlawane, N., Pan, H., Yan, M., et al. (2018). Prussian blue analogs for rechargeable batteries. Science 3, 110-133. doi: 10.1016/j.isci.2018.04.008

Wang, E., Chen, M., Liu, X., Liu, Y., Guo, H., Wu, Z., et al. (2018). Organic cross-linker enabling a $3 \mathrm{D}$ porous skeleton-supported $\mathrm{Na}_{3} \mathrm{~V}_{2}\left(\mathrm{PO}_{4}\right)_{3} /$ carbon composite for high power sodium-ion battery cathode. Small Methods 3:1800169. doi: 10.1002/smtd.201800169

Wang, E., Xiang, W., Rajagopalan, R., Wu, Z., Yang, J., Chen, M., et al. (2017). Construction of $3 \mathrm{D}$ pomegranate-like $\mathrm{Na}_{3} \mathrm{~V}_{2}\left(\mathrm{PO}_{4}\right)_{3} /$ conducting carbon composites for high-power sodium-ion batteries. J Mater. Chem. A 5, 9833-9841. doi: 10.1039/C7TA00153C

Wessells, C. D., Peddada, S. V., McDowell, M. T., Huggins, R. A., and Cui, Y. (2011). The effect of insertion species on nanostructured open framework hexacyanoferrate battery electrodes. J. Electrochem. Soc. 159, A98-103. doi: 10.1149/2.060202jes

Wong, L. L., Chen, H., and Adams, S. (2017). Design of fast ion conducting cathode materials for grid-scale sodium-ion batteries. Phys. Chem. Chem. Phys. 19, 7506-7523. doi: 10.1039/C7CP00037E

Wu, J., Song, J., Dai, K., Zhuo, Z., Wray, L. A., Liu, G., et al. (2017). Modification of transition-metal redox by interstitial water in hexacyanometalate electrodes for sodium-ion batteries. J. Am. Chem. Soc. 139, 18358-18364. doi: $10.1021 /$ jacs.7b10460

Xiao, P., Song, J., Wang, L., Goodenough, J. B., and Henkelman, G. (2015). Theoretical study of the structural evolution of a $\mathrm{Na}_{2} \mathrm{FeMn}$ $(\mathrm{CN})_{6}$ cathode upon $\mathrm{Na}$ intercalation. Chem. Mater. 27, 3763-3768. doi: 10.1021/acs.chemmater.5b01132

You, Y., Yao, H. R., Xin, S., Yin, Y. X., Zuo, T. T., Yang, C. P., et al. (2016). Subzerotemperature cathode for a sodium-ion battery. Adv. Mater. 28, 7243-7248. doi: 10.1002/adma.201600846

Zhang, Y., Malyi, O. I., Tang, Y., Wei, J., Zhu, Z., Xia, H., et al. (2017) Reducing the charge carrier transport barrier in functionally layer-graded electrodes. Angew. Chem. Int. Ed. 56, 14847-14852. doi: 10.1002/anie.2017 07883

Zhang, Y., Tang, Y., Deng, J., Leow, W. R., Xia, H., Zhu, Z., et al. (2019). Correlating the Peukert's constant with phase composition of electrode materials in fast lithiation processes. ACS Mater. Lett. 1, 519-525. doi: 10.1021/acsmaterialslett.9b00320

Zhu, T., Hu, P., Wang, X., Liu, Z., Luo, W., Owusu, K. A., et al. (2019). Realizing three-electron redox reactions in NASICON-structured $\mathrm{Na}_{3} \mathrm{MnTi}\left(\mathrm{PO}_{4}\right)_{3}$ for sodium-ion batteries. Adv. Energy Mater. 9:1803436. doi: 10.1002/aenm.201803436

Conflict of Interest: The authors declare that the research was conducted in the absence of any commercial or financial relationships that could be construed as a potential conflict of interest.

Copyright (c) 2020 Chen, Zhang, Xing and Tang. This is an open-access article distributed under the terms of the Creative Commons Attribution License (CC BY). The use, distribution or reproduction in other forums is permitted, provided the original author(s) and the copyright owner(s) are credited and that the original publication in this journal is cited, in accordance with accepted academic practice. No use, distribution or reproduction is permitted which does not comply with these terms. 